\title{
Preparation Process of Straw-based Activated Carbon for Uranium Wastewater Treatment
}

\author{
Hongtao Wang ${ }^{1,{ }^{*}}$, Rong Gui ${ }^{1}$, Wenxia $\mathrm{Zhu}^{1}$, Hankun lu ${ }^{1}$, Wenze Geng ${ }^{1}$, Jiayou Chen ${ }^{1}$, Lina $\mathrm{Wu}^{1}$ \\ ${ }^{1}$ University of South China, Hengyang, Hunan, China
}

\begin{abstract}
A process and equipment for preparing activated carbon from straw and its application in treating uranium wastewater are proposed. The $\mathrm{ZnCl}_{2}$-microwave carbonization method is adopted, which flexibly applies the wave absorbing ability of zinc element and greatly reduces the carbonization time. At the same time, the dehydrogenation of $\mathrm{ZnCl}_{2}$ limits the formation of coal tar and improves the carbon formation rate. Through citric acid modification, a functional group with specific adsorbability to uranyl ions is formed, which can produce high-efficiency complexation effect with uranyl ions and improve the adsorption capacity of uranyl ions. When the wastewater is mixed with other heavy metal elements, uranyl ions occupy the main adsorption sites, which can still maintain excellent targeted adsorption effect and can be targeted to treat uranyl ions in radioactive uranium wastewater. The key equipment in the process consists of cleaning and impurity removal components, raw material crushing components and carbonization components. The whole production process is completed in the same equipment. The optimum adsorption capacity $\left(A_{c}\right)$ of $4.98 \mathrm{mg} / \mathrm{g}$ and uranium adsorption rate $\left(A_{r}\right)$ of $99 \%$ were obtained by using soybean straw as raw material under the preparation conditions of $20 \% \mathrm{ZnCl}_{2}$ solution and $15 \%$ citric acid solution.
\end{abstract}

\section{Introduction}

In recent years, the nuclear industry has developed rapidly. As an important raw material for the nuclear industry, the demand for uranium is increasing daily [1]. However, a large amount of uranium-containing wastewater will be produced during the exploitation and utilization of uranium resources. If these uraniumcontaining wastewater is directly introduced into rivers, it will increase the toxicity of the water and destroy the ecological environment [2]. In addition, radionuclides can also be transferred to the human body through food such as rice, which would damage to human kidney, immune system, nervous system and fertility, and gene mutation and cancer [3]. Therefore, how to remove uranium pollution from the environment has become a concern. No matter from the perspective of uranium utilization and recovery, or from the perspective of ecological environment protection and human health, the treatment of uranium-containing wastewater is of great significance.

At present, the treatment methods of radioactive wastewater at home and abroad mainly include evaporation, chemical precipitation, ion exchange, adsorption, etc.[4-8]. Each method has its own advantages and disadvantages, and each method has different characteristics and application scope. Comparatively speaking, activated carbon adsorption method has attracted more and more attention in recent years due to its advantages of simple operation, abundant materials, mature process and wide application range [910]. The preparation of activated carbon is an important step in the treatment of uranium wastewater by activated carbon adsorption. The raw materials for preparing activated carbon mainly include wood, coal, coke, petroleum coke and fruit shell. However, facing the severe situation of shortage of raw materials, it has become the key to the preparation of activated carbon on finding new alternative raw materials. China is a large agricultural country, which produces 900 million tons of straw every year, and $17 \% \sim 26 \%$, that is, about 200 million tons of straw, will be directly burned in the open air, which not only wastes resources, but also causes serious environmental pollution problems. It is also a current research hotspot on how to reuse agricultural waste. In this paper, a new energy-saving special activated carbon production process is proposed. Its production process has the characteristics of simple operation and cleanness, is suitable for large-scale industrial production, and solves the problems of insufficient raw materials and complicated process faced by the current activated carbon preparation. This is not only conducive to the rational utilization of straw, but also provides high-quality activated carbon materials for uranium wastewater treatment.

\footnotetext{
* Corresponding author: 15573451647@,163.com
} 


\section{Materials and Methods}

\subsection{Materials}

The straw used in the experiment was selected from a soybean planting base in Hunan Province, and the main chemical composition inside the straw is shown in Table 1. Straw: soybean straw (taken from a soybean planting base in Hunan Province); Activator: Zinc Chloride (ZnCl2, analytically pure, Hunan Huihong Reagent Co., Ltd.); Modifier: Citric Acid (C6H8O7, analytically pure, Hunan Huihong Reagent Co., Ltd); Other reagents: Hydrochloric Acid, Anhydrous Ethanol, Nitrogen, Deionized Water, etc.

Table 1. Main Chemical Components of Soybean Straw

\begin{tabular}{|c|c|c|}
\hline \multicolumn{2}{|c|}{ Chemical composition } & Percentage (\%) \\
\hline \multicolumn{2}{|c|}{ Dry Matter(\%) } & 96.1 \\
\hline \multicolumn{2}{|c|}{ Ash $(\% \mathrm{DM})$} & 7.0 \\
\hline \multicolumn{2}{|c|}{ Crude protein $(\% \mathrm{DM})$} & 9.3 \\
\hline \multirow{4}{*}{$\begin{array}{c}\text { Fiber } \\
\text { Composition } \\
(\% \text { DM })\end{array}$} & Crude fiber & 29.3 \\
\hline & Cellulose & 32.9 \\
\hline & Lignin & 4.6 \\
\hline & Hemicellulose & 32.5 \\
\hline
\end{tabular}

\subsection{Experimental equipment}

This equipment is WZG-7 integrated activated carbon production equipment designed and developed by our team and with independent intellectual property rights. It has no special requirements on the initial form of raw materials, and the product has excellent adsorption effect on radioactive elements (including but not limited to uranyl, radium, europium, etc.). The equipment consists of three parts, namely, raw material cleaning equipment, raw material crushing equipment and carbonization treatment equipment. Its specific structure is shown in Figure 1.

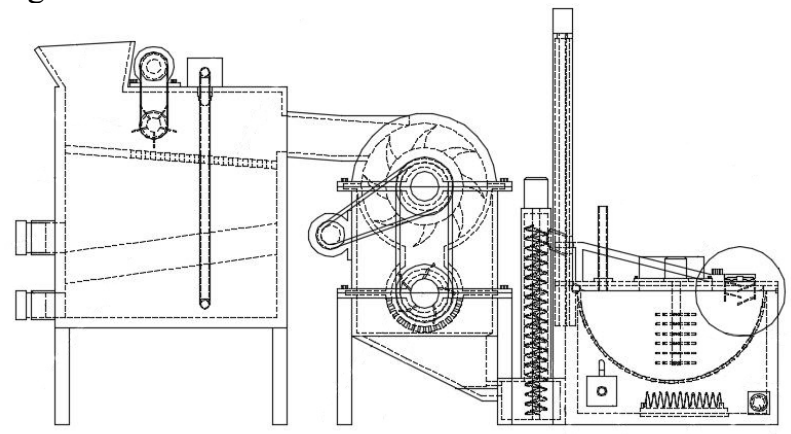

Fig.1. Internal Structure Diagram of the Equipment

\subsection{Preparation of the activated carbon}

1) Remove spoilage and miscellaneous leaves from that straw, and preliminarily clean up the silt.

2) Put the preliminarily treated straw into a cleaning equipment for washing. Straw enters the equipment through the feed port, and most of the straw moves on the water filter plate in the cleaning box under the action of the internal dial plate. The nozzle located on the inner side of the top wall of the cleaning box sprays highspeed water flow to clean the straw. Wastewater flows into the lower part through the water filter plate, and the cleaned straw moves to the upper part of the crushing box.

3) Crushing equipment. The crush wheel is driven by the external motor to crush the straw, and the crushed material with qualified particle size will fall into the material accumulation box through the lower screen, otherwise the crushed material will be repeatedly crushed until the particle size is qualified. The qualified crushed material in the material accumulation box is transported to the bottom of the storage box through the transportation pipe through the action of the worm, and the next treatment is carried out.

4) Heating and drying. The crushed objects located in the container can be dried and heated by closing the container cover and opening the bottom heater and the top air extraction fan. The drying temperature is 90 degrees and the time is 2 hours.

5) Activate operation, wherein that zinc chloride solution is injected into the contain box through the second pump body, and the stirring equipment is started to activate at the same time; or the nitrogen blowing pipe is opened to inject nitrogen into the inside of the contain box, so that the activation process is carried out in the nitrogen. The specific scheme design is listed in Table 2.

Table 2. Reagent concentration

\begin{tabular}{|c|c|c|}
\hline & $\begin{array}{c}\text { Zinc chloride } \\
\text { concentration } \\
\text { (mass fraction \%) }\end{array}$ & $\begin{array}{c}\text { Citric acid } \\
\text { concentration } \\
\text { (mass fraction \%) }\end{array}$ \\
\hline SM-1 & 10 & 10 \\
\hline SM-2 & 10 & 15 \\
\hline SM-3 & 10 & 20 \\
\hline SM-4 & 30 & 15 \\
\hline SM-5 & 20 & 15 \\
\hline SM-0 & Commercial coal-based activated carbon \\
\hline
\end{tabular}

6) Carbonize Operation. Open the liquid discharge port at the bottom of the container to empty the activated liquid zinc chloride solution, and then open the top suction fan and the bottom heater for drying. Subsequently, the microwave reactor on the inside of the top of the contain box is opened for carbonization, and the power of the microwave reactor is selectively adjusted according to the actual loading amount. The same step is preferably carried out in nitrogen atmosphere to prevent ashing and increase the carbon formation amount. 
7) After carbonization, $8 \%$ dilute hydrochloric acid is added through that second pump body to stir and heat, the temperature can be controlled within the range of 90100 degrees, acid washing is carried out, the time can be controlled to be 2 hours, and then the liquid is emptied.

8) Filling in $99 \%$ ethanol and stirring at room temperature, and then washing with deionize water until that $\mathrm{pH}$ value of the filtrate is neutral.

9) Filling citric acid solution respectively, opening that heater of the contain box to heat to 79 degrees, stirring and modifying under nitrogen atmosphere.

10) Washing with deionized water, drying, and storing.

\subsection{Adsorption test}

Raw materials: uranium standard reserve solution (reference reagent U3O8, CNNC 272 Co., Ltd.), commercial activated carbon (powder), soybean strawbased activated carbon, iodine standard solution, sodium thiosulfate, hydrochloric acid, etc.

Equipment: Ultraviolet visible spectrophotometer (Model: T6 New Century, produced by Beijing Purkinje Genera Instrument Co., Ltd.); Fourier transform infrared spectrum analyzer (Spectrum One produced by PerkinElmer)); S-4700 scanning electron microscope (Hitachi), etc.

\subsubsection{Determination of iodine adsorption value of activated carbon}

According to the Specification for Determination of Iodine Adsorption Value of Wooden Activated Carbon Test Method in China (GB/T 12496.8-2015), take out the refined oil tea shell-based activated carbon which has passed through the standard sieve with a pore diameter of $74 \mu \mathrm{m}$ after being dried, weigh $0.50 \mathrm{~g}$ accurately, move it into a $250 \mathrm{~mL}$ conical flask with a grinding mouth, fill in $10 \mathrm{~mL}$ of $5 \%$ hydrochloric acid, boil it on an electric furnace for 30 s after being fully mixed, fill in $50 \mathrm{~mL}$ of iodine standard solution with a concentration of $0.1 \mathrm{~mol} / \mathrm{L}$ after cooling, put the bottle cap on the bottle, shake it in a shaking table, take it out and filter after $15 \mathrm{~min}$. In conical flask, $10 \mathrm{~mL}$ filtrate and $100 \mathrm{~mL}$ double distilled water were accurately removed by pipette respectively. It is titrated with $0.1 \mathrm{~mol} / \mathrm{L}$ sodium thiosulfate standard solution. During titration, when the color of the solution becomes light, $2 \mathrm{~mL}$ starch indicator should be added dropwise until the titration becomes colorless.

Formula for calculating iodine adsorption value were shown as equation (1) to (3).

$$
\begin{gathered}
A=\frac{X}{M} \cdot D \\
\frac{X}{D}=\frac{5\left(10 c_{1}-1.2 c_{2} V_{2}\right) \times 126.93}{m} \\
c=\frac{c_{2} V_{2}}{10}
\end{gathered}
$$

Where: $A$ is iodine adsorption value, $\mathrm{mg} / \mathrm{g} ; X / M$ is the iodine adsorption value per unit mass of activated carbon, $\mathrm{mg} / \mathrm{g} ; D$ is a correction factor; $c_{1}$ is the concentration of the standard solution of iodine, mol/L; $c_{2}$ is the concentration of the standard solution of sodium sulphate, $\mathrm{mol} / \mathrm{L} ; m$ is the amount of activated carbon used, $\mathrm{g} ; c$ is the concentration of iodine in the remaining filter, $\mathrm{mol} / \mathrm{L} ; 126.93$ is the unit of iodine mole mass.

\subsubsection{Experiment on adsorption of uranium (VI) by activated carbon}

Use pipette to accurately measure $50 \mathrm{~mL}$ of uranium standard solution with appropriate mass concentration in a $250 \mathrm{~mL}$ conical flask. Adopt dilute hydrochloric acid and $\mathrm{NaOH}$ solution to adjust the $\mathrm{pH}$ value of the solution, then add appropriate amount of activated carbon; The adsorption is carried out in a shaking table with a temperature of $25^{\circ} \mathrm{C}$ and a rotating speed of $200 \mathrm{r} / \mathrm{min}$. After adsorption, the surface clear liquid was filtered, and the mass concentration of uranium was determined by ultraviolet spectrophotometer, and the adsorption capacity $\left(A_{c}\right)$ and adsorption rate $\left(A_{r}\right)$ were selected to evaluate the adsorption performance of activated carbon for uranium (VI).

\section{Results \& Discussion}

The adsorption performance of the finished carbon is tested, and the specific experimental results are shown in Table 3.

Table 3. Characterization Data of Activated Carbon under Different Conditions

\begin{tabular}{|c|c|c|c|c|}
\hline & $\begin{array}{c}\text { Uranium } \\
\text { adsorption } \\
\text { value } \\
\text { mg•g }^{-1}\end{array}$ & $\begin{array}{c}\text { Iodine } \\
\text { adsorption } \\
\text { value } \\
\text { mg・g }^{-1}\end{array}$ & $\begin{array}{c}\text { Specific } \\
\text { surface } \\
\text { area } \\
\mathbf{m}^{2} \bullet \mathbf{g}^{-1}\end{array}$ & $\begin{array}{c}\text { Yield } \\
\mathbf{( \% )}\end{array}$ \\
\hline SM-0 & 1.32 & 657.3 & 1023.4 & - \\
\hline SM-1 & 4.13 & 831 & 909.4 & 49.1 \\
\hline SM-2 & 4.67 & 920.3 & 1235.4 & 41 \\
\hline SM-3 & 4.51 & 952.2 & 1356.9 & 39.2 \\
\hline SM-4 & 4.21 & 802.3 & 956.7 & 37.5 \\
\hline SM-5 & 4.98 & 918.4 & 1204.6 & 41.6 \\
\hline
\end{tabular}

The results showed that when $\mathrm{pH}$ was 6 , initial uranium concentration was $5 \mathrm{mg} / \mathrm{L}$, activated carbon dosage was $1 \mathrm{~g} / \mathrm{L}$ and adsorption time was $8 \mathrm{~h}$, the filtrate was taken 10 times in the adsorption column, and the uranyl ion concentration in the filtrate was measured by ultraviolet spectrophotometer, and the data were recorded. The best adsorption capacity $\left(A_{c}\right)$ was 4.98 $\mathrm{mg} / \mathrm{g}$ and the uranium adsorption rate $\left(A_{r}\right)$ was $99 \%$. Compared with common commercial activated carbon, the activated carbon produced by this process has 
extremely high adsorption and removal capacity for uranium.

Zinc chloride activation combined with microwave carbonization process greatly improves carbonization efficiency by utilizing the ability of zinc element to absorb waves. Compared with the traditional method of long-term high-temperature sintering carbonization in a high-temperature environment of several hundred degrees Celsius, the microwave carbonization stage only needs a short time of 2-3 minutes, saving a large amount of energy, and achieving high efficiency, energy saving and high quality.

Dehydrogenation of zinc chloride limits the formation of tar and can improve the subsequent charring rate. Zinc chloride molecules are impregnated into the interior of carbon to play a skeleton role. Carbon polymers are carbonized and deposited on the skeleton. When zinc chloride is washed away with acid and hot water, it is conducive to the formation of porous activated carbon with huge surface.

Citric acid modification is used to form a functional group with specific adsorption for uranyl ions, which can produce efficient complexation effect with uranyl ions and improve the adsorption effect for uranyl ions. When other heavy metal elements are mixed in the wastewater, uranyl ions occupy the main adsorption sites, which can still maintain good targeted adsorption effect and is conducive to targeted treatment of uranium in radioactive uranium wastewater.

\section{Conclusion}

This project proposes a preparation process of special activated carbon, which uses agricultural waste straw as raw material and is specially used for radionuclide (uranium) pollution treatment, and draws the following conclusions.

1) When the mass concentration of zinc chloride activator is $20 \%$ and the concentration of citric acid modifier is $15 \%$, the specific surface area of the prepared straw-based activated carbon is as high as $1204.6 \mathrm{~m} 2 / \mathrm{g}$ and the iodine adsorption value is $918.4 \mathrm{mg} / \mathrm{g}$.

2) Under the conditions of $\mathrm{pH}$ value 6 , dosage $1 \mathrm{~g} / \mathrm{L}$, initial uranium concentration $5 \mathrm{mg} / \mathrm{L}$ and adsorption time $8 \mathrm{~h}$, the optimum adsorption capacity $\left(A_{c}\right)$ of straw-based activated carbon is $4.98 \mathrm{mg} / \mathrm{g}$, and the uranium adsorption rate $\left(A_{r}\right)$ is $99 \%$.

3) The activated carbon has excellent adsorption effect on uranium, because the proportion of carboxyl groups on the surface of the modified activated carbon after citric acid increases, and functional groups such as carboxyl groups and the like undergo complexation reaction with uranyl ions in the adsorption process.

\section{References}

1. Gao Junkai, Gu Ping, Zhang Guangguang, Gao Xin, Hou Lian. The research progress of adsorption treatment of low-concentration uranium-containing wastewater[J]. Chinese engineering science.,2014,16(07):73-78.
2. Huang Qijin. J. Chemical Engineering and Equipment was studied by comparison method of uranium-containing wastewater treatment technology,2018(06):269-272.

3. Hu Eming,Shao Eryan, Zhao Jing. Advances in the research of uranium-containing wastewater treatment technology $[\mathrm{J}]$. Hunan Journal of Ecological Sciences.,2016,3(01):42-48.

4. Wei Guangzhi, $\mathrm{Xu}$ Lechang. The treatment technology of low-concentration uraniumcontaining wastewater and its research progress [J]. Uranium mining and metallurgy.,2007(02):90-95.

5. A.K. Darban, A. Shahedi, F. Taghipour, A. Jamshidi-Zanjani,A review on industrial wastewater treatment via electrocoagulation processes, Current Opinion in Electrochemistry,2020.

6. Haiyang Liu, Jianlong Wang,Treatment of radioactive wastewater using direct contact membrane distillation,Journal of Hazardous Materials, Volume 261,2013.

7. Fida Tibi, Amine Charfi, Jinwoo Cho, Jeonghwan Kim,Fabrication of polymeric membranes for membrane distillation process and application for wastewater treatment: Critical review,Process Safety and Environmental Protection,Volume 141,2020.

8. Phumza V. Tikilili, Evans M. NkhalambayausiChirwa,Characterization and biodegradation of polycyclic aromatic hydrocarbons in radioactive wastewater,Journal of Hazardous Materials, Volume 192, Issue 3,2011.

9. Zhang Wei. Adsorption method separation uranium introduction $[\mathrm{J}]$. private science and technology.,2017(02):46.

10. Zuo Tianming, Zuo Weiming, Li Jinlian. Activated carbon adsorption treatment of uranium-containing radioactive wastewater[J]. Sichuan Geology,2015,35(03):473-475. 